\title{
ON CURVATURE DECAY IN EXPANDING COSMOLOGICAL MODELS
}

\author{
HANS RINGSTRÖM
}

\begin{abstract}
Consider a globally hyperbolic cosmological spacetime. Topologically, the spacetime is then a compact 3-manifold in cartesian product with an interval. Assuming that there is an expanding direction, is there any relation between the topology of the 3-manifold and the asymptotics? In fact, there is a result by Michael Anderson, where he obtains relations between the longtime evolution in General Relativity and the geometrization of 3-manifolds. In order to obtain conclusions however, he makes assumptions concerning the rate of decay of the curvature as proper time tends to infinity. It is thus of interest to find out if such curvature decay conditions are always fulfilled. We consider here the Gowdy spacetimes, for which we prove that the decay condition holds. However, we observe that for general Bianchi VIII spacetimes, the curvature decay condition does not hold, but that some aspects of the expected asymptotic behaviour are still true.
\end{abstract}

\section{INTRODUCTION}

The objects of study in this paper are cosmological spacetimes. We shall assume them to be globally hyperbolic, so that topologically, they are of the form $I \times M$, where $M$ is a compact 3-manifold. We shall also only consider spacetimes which have one expanding direction, i.e. there is one time direction in which spacetime is causally geodesically complete. The question is then, what is the relationship between the asymptotic behaviour and the topology of the compact Cauchy surfaces? Anderson, Fischer and Moncrief have written several papers on the subject, see [2] and [7] and the references cited therein. In the current paper, we are concerned with questions raised in [2] regarding the relationship between the asymptotics and geometrization. The special case of interest here is when one has a globally hyperbolic vacuum spacetime foliated by compact constant mean curvature (CMC) hypersurfaces, though in the case of Gowdy, we shall also be interested in another geometrically defined foliation. We shall assume that $\sigma(\Sigma) \leq 0$ for any CMC hypersurface (for a definition of the $\sigma$-constant of a compact 3-manifold, see [1]) or, in other words, that $\Sigma$ does not admit a metric of positive scalar curvature, see [2]. Furthermore, we shall assume that the range of the mean curvatures attained in the foliation exhausts the interval $(-\infty, 0)$ and that the spacetime is future causally geodesically complete. In fact, we shall only be interested in the expanding direction, so it is enough if we the foliation exhausts the interval $\left[H_{0}, 0\right)$ for some $H_{0}<0$, and sometimes future causal geodesic completeness will be a consequence of other assumptions. In this setting we wish to consider the behaviour of the geometry induced on the leaves of the foliation as proper time tends to infinity. Let us recall some definitions from [2]. 
Definition 1.1. Let $\Sigma$ be a closed, oriented and connected 3-manifold, satisfying $\sigma(\Sigma) \leq 0$. A weak geometrization of $\Sigma$ is a decomposition of $\Sigma$,

$$
\Sigma=H \cup G,
$$

where $H$ is a finite collection of complete connected hyperbolic manifolds of finite volume embedded in $\Sigma$ and $G$ is a finite collection of connected graph manifolds embedded in $\Sigma$. The union is along a finite collection of embedded tori $\mathcal{T}=\cup T_{i}$, $\mathcal{T}=\partial H=\partial G$. A strong geometrization of $\Sigma$ is a weak geometrization as above, for which each torus $T_{i}$ in $\mathcal{T}$ is incompressible in $\Sigma$, i.e. the inclusion of $T_{i}$ into $\Sigma$ induces an injection of fundamental groups.

For more details concerning the terminology, we refer to [2] and the references cited therein. Graph manifolds are built by gluing together Seifert fibred spaces along toral boundary components. Since we shall only be concerned with Seifert fibred 3 -manifolds in this paper, the details of these constructions are not of any greater importance here. Let us however define the concept Seifert fibred space.

Definition 1.2. A 3-manifold is said to be a Seifert fibred space if it satisfies the following two conditions:

(1) It can be written as a disjoint union of circles.

(2) Each circle fibre has an open neighbourhood $U$ satisfying:

- $U$ can be written as a disjoint union of circle fibres,

- $U$ is isomorphic either to a solid torus or a cylinder where the ends have been identified after a rotation by a rational angle.

When we say that $U$ is isomorphic to a solid torus, we mean that $U$ is diffeomorphic to a solid torus and that the circle fibres of $U$ are mapped to the natural circle fibres of the solid torus under the diffeomorphism.

Note that there are different definitions of Seifert fibred spaces in the literature. In particular, our definition coincides with the original definition by Seifert but not with that of Scott [14].

Since the geometry on the leaves of the foliation becomes more and more flat, it is natural to rescale the metric in some way. Following [2], we shall use the proper time distance to a fixed Cauchy surface in order to do so. Let $\Sigma$ be a fixed Cauchy surface and define, for an arbitrary spacetime point $p$,

$$
\hat{t}(p)=\sup _{\gamma} \int_{0}^{1}\left[-\left\langle\gamma^{\prime}, \gamma^{\prime}\right\rangle\right]^{1 / 2} d s
$$

where the supremum is taken over timelike curves $\gamma$ with $\gamma(0) \in \Sigma$ and $\gamma(1)=p$ and $\langle\cdot, \cdot\rangle$ denotes inner product with respect to the spacetime metric. We also define

$$
\hat{t}\left(\Sigma^{\prime}\right)=\sup _{p \in \Sigma^{\prime}} \hat{t}(p)
$$

for a Cauchy surface $\Sigma^{\prime}$. Let the leaves of the foliation be indexed by a parameter $s$. In the case of a CMC foliation, the parameter can be chosen to be the mean curvature of the corresponding leaf, and in the case of Gowdy, the parameter will be the so called areal time coordinate. We are interested in the interval $\left[s_{0}, s_{\max }\right)$, where $s_{0}$ corresponds to some arbitrary initial hypersurface (filling the role of $\Sigma$ above) and $s_{\max }$ corresponds to infinite expansion, i.e. $s_{\max }=0$ in the CMC case 
and $s_{\max }=\infty$ in the case of the areal time coordinate in Gowdy. Let $\hat{g}_{s}$ be the Riemannian metric induced on the leaf $\Sigma_{s}$ by the spacetime metric and define

$$
g_{s}=\hat{t}^{-2}\left(\Sigma_{s}\right) \hat{g}_{s}
$$

The following weak asymptotics problem was raised in [2]. Suppose that $\Sigma$ is a closed, oriented, connected 3-manifold with $\sigma(\Sigma) \leq 0$. Suppose further that the vacuum spacetime is future causally geodesically complete and that the CMC foliation exhausts the future development. Then for any sequence $s_{i} \rightarrow s_{\max }$, the slices $\left(\Sigma_{s_{i}}, g_{s_{i}}\right)$ have a subsequence asymptotic to a weak geometrization of $\Sigma$. More precisely, there should be a division of $\Sigma$ as in (1) and on the region $H$, the metrics $g_{s_{i}}$ should converge to complete hyperbolic metrics of finite volume, while on $G$, the metrics collapse the graph manifold with bounded curvature. When we say that a region collapses we mean that the injectivity radius of that region converges to zero. If a region collapses even though the curvature remains bounded, we shall say that is collapses in the sense of Cheeger-Gromov.

This conjecture should be compared with the work of Andersson and Moncrief [3], Choquet-Bruhat and Moncrief [4] and Fischer and Moncrief [7]. In [3], the authors considered the future development of perturbations of spatially compact variants of the $k=-1$ Friedmann-Robertson-Walker vacuum spacetime. They proved that the future development is covered by CMC hypersurfaces exhausting the maximal range, and that it is future causally geodesically complete. Furthermore, the rescaled metric on the spatial hypersurfaces was shown to converge to the hyperbolic one. In [4], the authors considered Cauchy surfaces that have the topology of a trivial circle bundle over a higher genus surface and they restricted their attention to small, polarized, $U(1)$-symmetric data. They proved that the future development is foliated by CMC hypersurfaces exhausting the maximal range. Furthermore, they stated that causal geodesic completeness should hold, though they did not prove it. However, this was shown for a larger class of spacetimes in [5], a paper which extends the results of [4] to the non-polarized case, using the results of [6]. Finally, they showed that the Cauchy surfaces should undergo a Cheeger-Gromov type collapse. In [7], some known spatially homogeneous examples were studied and the expected behaviour was confirmed. Note that in all the cases mentioned above, either $H=\varnothing$ or $G=\varnothing$ in the division (1). The reason for this is the fact that all results, as far as we are aware, can be divided into the category of small data results and the category of results for a situation in which there is symmetry. The small data category may seem to be more general, but since it presupposes the existence of a symmetric solution around which to perturb, it is not more general in terms of spatial topology. In other words, all results known require the spatial manifold to allow a highly symmetric metric, and this reduces the number of allowed spatial topologies.

In [2], the following statement was proved. Consider a spacetime which is the maximal development of vacuum initial data, with $\sigma(\Sigma) \leq 0$, where $\Sigma$ is the initial hypersurface, and assume that it is foliated to the future by CMC hypersurfaces exhausting the maximum range. Assume furthermore that the curvature satisfies

$$
|R|(p)+\hat{t}(p)|\nabla R|(p) \leq \frac{C}{\hat{t}^{2}(p)},
$$


where $|R|^{2}$ is defined as the sum of the squares of the components of the Riemann curvature tensor with respect to an orthonormal frame, where the timelike unit vector in the frame is the future oriented normal to the foliation (the definition of $|\nabla R|^{2}$ is similar). Then the spacetime is future causally geodesically complete and, for any sequence $s_{i} \rightarrow s_{\max }$, the slices $\left(\Sigma_{s_{i}}, g_{s_{i}}\right)$ have a subsequence asymptotic to a weak geometrization.

Due to this theorem, it is of interest to analyze how curvature decays in expanding cosmological spacetimes. In the following, we shall only consider whether the estimate

$$
|R|(p) \leq \frac{C}{\hat{t}^{2}(p)},
$$

holds or not. In the case of Gowdy, it turns out that such an estimate holds, at least relative to the foliation defined by the areal time coordinate. In the case of locally rotationally symmetric Bianchi VIII, the estimate also holds, but it turns out that for general Bianchi VIII it does not. In that case $\hat{t}(p) \ln \hat{t}(p)|R|(p)$ converges to a positive number as $p$ tends to a point in the infinite future. In fact, in the case of general Bianchi VIII, one does not get a better estimate even if one considers the Kretschmann scalar

$$
\kappa=R_{\alpha \beta \gamma \delta} R^{\alpha \beta \gamma \delta} .
$$

It is then of interest to consider the Ricci curvature of $g_{s_{i}}$. It turns out that in general, the Ricci curvature does not have any better decay, but that there is a time sequence such that one does get the expected decay. This time sequence corresponds to the metric being locally rotationally symmetric. Concerning the topology, we have the following results. In the case of Gowdy, the topology is $T^{3}$, and after rescaling the 3 -tori collapse along 2-tori. In the Bianchi VIII case, the topology is that of a non-trivial circle bundle over a higher genus surface. After rescaling one obtains the conclusion that the length of the circle fibers converges to zero.

1.1. Gowdy spacetimes. The Gowdy spacetimes is a class of vacuum spacetimes with a two dimensional group of isometries. Of the spatial topologies compatible with the symmetry requirements, only $T^{3}$ is expected to be compatible with infinite expansion. For this reason, we shall only be interested in such a spatial topology in this paper. There are natural conditions defining the Gowdy spacetimes, see [12] and references therein, but we shall not write them down here. For the purposes of the present paper, a Gowdy $T^{3}$ spacetime is defined as a Lorentz manifold $\mathbb{R}_{+} \times T^{3}$, where $\mathbb{R}_{+}=(0, \infty)$, with metric

$$
g=t^{-1 / 2} e^{\lambda / 2}\left(-d t^{2}+d \theta^{2}\right)+t\left[e^{P} d \sigma^{2}+2 e^{P} Q d \sigma d \delta+\left(e^{P} Q^{2}+e^{-P}\right) d \delta^{2}\right],
$$

where $P, Q$ and $\lambda$ only depend on $t$ and $\theta$, satisfying Einstein's vacuum equations. In terms of $P, Q$ and $\lambda$, the equations are

$$
\begin{aligned}
P_{t t}+\frac{1}{t} P_{t}-P_{\theta \theta}-e^{2 P}\left(Q_{t}^{2}-Q_{\theta}^{2}\right) & =0 \\
Q_{t t}+\frac{1}{t} Q_{t}-Q_{\theta \theta}+2\left(P_{t} Q_{t}-P_{\theta} Q_{\theta}\right) & =0,
\end{aligned}
$$


and

$$
\begin{aligned}
& \lambda_{t}=t\left[P_{t}^{2}+P_{\theta}^{2}+e^{2 P}\left(Q_{t}^{2}+Q_{\theta}^{2}\right)\right] \\
& \lambda_{\theta}=2 t\left(P_{\theta} P_{t}+e^{2 P} Q_{\theta} Q_{t}\right) .
\end{aligned}
$$

The time coordinate $t$ appearing in (5) is called the areal time coordinate. The reason for this is that the area of the two torus given by fixing $t$ and $\theta$ is $t$. On the other hand, the trace of the second fundamental form need not be constant on the hypersurfaces of constant $t$. One might then naively expect this to approximately be the case asymptotically. However, there are metrics of the form (5) such that there is a time sequence $t_{k} \rightarrow \infty$ with the property that the quotient of the maximum and the minimum of $\left|\operatorname{tr} k_{t_{k}}\right|$ tends to infinity, where $k_{t_{k}}$ is the second fundamental form of the hypersurface defined by $t=t_{k}$. We refer the reader to [13] for a proof of this fact. Thus there is certainly a difference between the CMC foliation and the areal time coordinate foliation. Since most of the analysis concerning Gowdy spacetimes has been carried out in the areal time coordinate and since this coordinate has a natural geometric definition, we shall however only consider this choice here. In the end we are interested in getting estimates for the curvature. In [12], we analyzed the asymptotics of solutions to (6)-(7). However, the analysis was not complete. In particular, [12] only contains estimates of the first derivatives of $P$ and $Q$, and this is not sufficient for computing curvature. The first step is to remedy this situation.

Theorem 1. Consider a solution to (6)-(7). Then

$$
\left\|\left(\partial_{\theta}^{k} \partial_{t} P\right)^{2}+\left(\partial_{\theta}^{k+1} P\right)^{2}+e^{2 P}\left[\left(\partial_{\theta}^{k} \partial_{t} Q\right)^{2}+\left(\partial_{\theta}^{k+1} Q\right)^{2}\right]\right\|_{C^{0}\left(S^{1}, \mathbb{R}\right)} \leq C_{k} \frac{(\ln t)^{2 k}}{t}
$$

for $t \geq 2$ and $k \geq 0$.

Remark. The above estimates together with the equations (6)-(7) yield estimates for the higher order derivatives involving an arbitrary number of time derivatives. In the polarized case, i.e. when $Q=0$, there is an improved estimate. In fact, one does not need the logarithms. To see this, note that the case $k=0$ of (10) was proved in [12] and that in the polarized case, the equation remains the same under differentiation with respect to $\theta$.

The proof is to be found at the beginning of section 2. Define the proper time distance between the hypersurfaces defined by $t_{0}$ and $t$ to be $\tau\left(t_{0}, t\right)$, cf. (18). Then the decay estimate for the curvature is as follows.

Theorem 2. Consider a metric of the form (5), where $P, Q$ and $\lambda$ satisfy (6)-(9). Assume furthermore that $P$ and $Q$ are not both independent of $\theta$ for all $t$. Then for every $t_{0}>0$, there is a positive constant $C\left(t_{0}\right)$ and a $T\left(t_{0}\right)$ such that for $t \geq T\left(t_{0}\right)$,

$$
|R|(t) \leq C\left(t_{0}\right) \tau^{-2}\left(t_{0}, t\right),
$$

where $|R|$ is defined with respect to the areal time coordinate foliation.

Remark. When considering metrics of the form (5), the spatially homogeneous solutions have a special type of behaviour. In particular, if there is some spatial variation, $\lambda$ tends to infinity linearly, but if there is no spatial variation, $\lambda$ tends to infinity logarithmically, cf. [12]. Since $P$ cannot grow faster than logarithmically and $Q$ cannot grow faster than polynomially, cf. [12], it is clear that in the spatially inhomogeneous case, the factor in front of $-d t^{2}+d \theta^{2}$ tends to infinity exponentially whereas all the other factors tend to infinity at worst polynomially. In other 
words, all the expansion is in the factor in front of $-d t^{2}+d \theta^{2}$. In the spatially homogeneous case, there is however no such clear distinction between the different factors, since $\lambda$ tends to infinity logarithmically. For this reason we focus on the spatially inhomogeneous case and leave the homogeneous case to the reader.

The proof is to be found at the end of section 2. Finally, let us say something about the rescaled Riemannian metric on the hypersurfaces of constant areal time. The proof is also to be found at the end of section 2 .

Proposition 1. Consider a metric of the form (5), where $P, Q$ and $\lambda$ satisfy (6)(9). Assume furthermore that $P$ and $Q$ are not both independent of $\theta$ for all $t$. Let $\hat{g}_{t}$ be the Riemannian metric induced on the hypersurface of constant areal time $t$, and let $g_{t}=\hat{g}_{t} / \tau^{2}\left(t_{0}, t\right)$. Then $g_{t}$ is a metric on $T^{3}$, which can be written

$$
g_{t}=f_{1}(t, \theta) d \theta^{2}+f_{2}(t, \theta) d \delta^{2}+f_{3}(t, \theta) d \delta d \sigma+f_{4}(t, \theta) d \sigma^{2} .
$$

The family $f_{1}(t, \cdot)$ of functions is bounded in $C^{1}$ and from below by a positive constant, for $t \geq t_{0}+1$. For $i \geq 2, k \geq 0$ and $t \geq t_{0}+1$, we have the following estimate,

$$
\left\|f_{i}(t, \cdot)\right\|_{C^{k}} \leq C_{k} \frac{\left\{\ln \left[1+\tau\left(t_{0}, t\right)\right]\right\}^{\alpha_{k}}}{\tau^{2}\left(t_{0}, t\right)}
$$

where $\alpha_{k}$ and $C_{k}$ are positive constants.

Remark. By the conclusions of the proposition and the Arzela-Ascoli theorem, there is, for any time sequence $t_{k} \rightarrow \infty$, a subsequence such that $f_{1}\left(t_{k}, \cdot\right)$ converges to a positive continuous function (the limit function will of course be Lipschitz). Furthermore, it is clear that the metric collapses in the two-torus direction defined by $\delta$ and $\sigma$. Finally, if it were possible to improve the estimate (10) in such a way that the logarithms do not occur, $f_{1}(t, \cdot)$ would be bounded in any $C^{k}$ norm for $t \geq t_{0}+1$. In particular, in the polarized Gowdy case, we have such bounds.

1.2. Bianchi VIII. For proofs of the statements made below, we refer the reader to [11] and the references cited therein. We define Bianchi VIII spacetimes in terms of initial data. Bianchi VIII initial data are given by $(G, g, k)$, where $G$ is a Lie group of Bianchi type VIII (to be defined below), $g$ is a left invariant metric, $k$ is a left invariant symmetric two tensor and $g$ and $k$ satisfy the constraint equations. In practice, $G$ can be assumed to be the universal covering group of $\operatorname{Sl}(2, \mathbb{R})$. However, in general, a Lie group $G$ is said to be of Bianchi type VIII if it has a basis $e_{i}^{\prime}$ of the Lie algebra satisfying

$$
\left[e_{i}^{\prime}, e_{j}^{\prime}\right]=\gamma_{i j}^{k} e_{k}^{\prime}
$$

with $\gamma_{i j}^{k}=\epsilon_{i j l} n^{l k}$, where $\epsilon_{i j l}$ is antisymmetric in all its indices, $\epsilon_{123}=1$, and $n^{l k}$ is diagonal with diagonal components $n_{i}$ such that $n_{1}<0$ and $n_{2}, n_{3}>0$. Given initial data, there is a basis $e_{i}^{\prime}$ satisfying the conditions of the previous sentence such that $g$ is orthonormal with respect to this basis and $k$ is diagonal. We call such a basis a canonical basis. Such bases are not unique, but it turns out that $e_{1}^{\prime}$ is well defined up to a sign. Let $k_{i}=k\left(e_{i}^{\prime}, e_{i}^{\prime}\right)$. Then the initial data are said to be of NUT type if $k_{2}=k_{3}$ and $n_{2}=n_{3}$. Given initial data, one can construct a globally hyperbolic Lorentz manifold $(I \times G, \bar{g})$, where $I$ is an open interval and $\bar{g}$ 
is of the form

$$
\bar{g}=-d t^{2}+\sum_{i=1}^{3} a_{i}^{2}(t) \xi^{i} \otimes \xi^{i}
$$

where the $\xi^{i}$ are the duals of $e_{i}^{\prime}$, a canonical basis, and $a_{i}(0)=1$. Finally $\operatorname{Ric}[\bar{g}]=0$ and the Riemannian metric and the second fundamental form induced on $\Sigma=$ $\{0\} \times G$ by $\bar{g}$ are given by $g$ and $k$, after identifying $G$ with $\Sigma$ in the obvious way. The development is future causally geodesically complete and independent of the canonical basis chosen. If the data are not of NUT type, the development is $\mathrm{C}^{2}$ inextendible, in fact, the Kretschmann scalar (4) is unbounded to the past, cf. [8]. Finally, if the data are of NUT type, $a_{2}(t)=a_{3}(t)$ for all $t$.

We can, without loss of generality, assume $G$ to be $\tilde{\mathrm{Sl}}(2, \mathbb{R})$, the universal covering group of $\operatorname{Sl}(2, \mathbb{R})$. Since $\tilde{\mathrm{Sl}}(2, \mathbb{R})$ is diffeomorphic to $\mathbb{R}^{3}$, it is of interest to know when the geometry allows compactifications of the spatial hypersurfaces. In [11] we showed that if $\Gamma$ is a free and properly discontinuous subgroup of the isometry group of the initial data $(G, g, k)$, then $\{\operatorname{Id}\} \times \Gamma$ is a free and properly discontinuous subgroup of the isometry group of the development. By taking the quotient, we thus get developments such that the corresponding CMC hypersurfaces have topology $G / \Gamma$. Furthermore, the compact manifold $G / \Gamma$ must be Seifert fibred and $e_{1}^{\prime}$ corresponds to the Seifert fibre direction. We also proved that $a_{1}=l_{0}+O\left(t^{-1}\right)$ in the NUT case and $a_{1}(t)=c_{0}(\ln t)^{1 / 2}[1+O(\ln \ln t / \ln t)]$ in the non-NUT case. Furthermore $a_{i}(t) / t \rightarrow \alpha_{i}>0$ for $i=2,3$. Thus, after rescaling, the Seifert fibred spaces collapse as expected. Note that for each $p>1$, there is a subgroup $\Xi_{p}$ of $\tilde{\mathrm{Sl}}(2, \mathbb{R})$ such that the quotient of $\tilde{\mathrm{Sl}}(2, \mathbb{R})$ by $\Xi_{p}$ (when $\Xi_{p}$ is viewed as a group of isometries by acting on the left) is diffeomorphic to the unit tangent bundle of a compact orientable surface of genus $p$ with respect to some hyperbolic metric. Thus all initial data allow infinitely many different compactifications. However, the following holds.

Theorem 3. Consider a Bianchi VIII spacetime. If it is of NUT type, there are constants $c_{0}, c_{1}>0$ and a $T>0$, such that

$$
c_{0} t^{-3} \leq|R|(t) \leq c_{1} t^{-3}
$$

for all $t \geq T$. If it is of non-NUT type, there is a constant $c_{0}>0$ such that

$$
\lim _{t \rightarrow \infty} t \ln t|R|(t)=c_{0} .
$$

Furthermore, there are constants $c_{i}>0$ and sequences $t_{i, k} \rightarrow \infty, i=1,2$, such that

$$
\lim _{k \rightarrow \infty} t_{i, k}^{2}\left(\ln t_{i, k}\right)^{2} \kappa\left(t_{i, k}\right)=(-1)^{i} c_{i},
$$

where $\kappa$ is defined in (4).

The proofs of this result and the next are to be found in section 3 .

One can then ask the question if the Ricci curvature of the spatial hypersurfaces behaves better. This turns out not to be the case in general, but there is in fact a time sequence along which it behaves well.

Proposition 2. Consider a Bianchi VIII spacetimes which is not of NUT type. Then there are time sequences $t_{i, k} \rightarrow \infty, i=1,2$, and positive constants $c_{i}$ such 
that

$$
\lim _{k \rightarrow \infty} t_{1, k}^{2}\left(\ln t_{1, k}\right)^{2}\left(R_{i j} R^{i j}\right)\left(t_{1, k}\right)=c_{1}, \quad t_{2, k}^{4}\left(R_{i j} R^{i j}\right)\left(t_{2, k}\right) \leq c_{2},
$$

where the last inequality is valid for all $k$, and $R_{i j}(t)$ denotes the Ricci tensor of the spatial hypersurface of homogeneity defined by $t$, with metric induced by $\bar{g}$.

Remark. The time sequence $t_{2, k}$ corresponds to the induced Riemannian metric being locally rotationally symmetric. Due to the existence of the sequence $t_{1, k}$, the conjecture embodied in the weak asymptotics problem is not correct.

\section{Curvature estimates for Gowdy}

The expanding direction of Gowdy spacetimes was considered in [12]. The leading order behaviour for the functions $P, Q$ and $\lambda$ was sorted out and (10) was proved to hold for $k=0$. In this paper, we are interested in the behaviour of curvature quantities, and thus we need to concern ourselves with the asymptotic behaviour of higher order derivatives.

Proof of Theorem 1. By [12], we know that the conclusion holds for $k=0$. Define

$$
\begin{aligned}
\mathcal{A}_{k, \pm} & =\frac{t}{2}\left[\left(\partial_{\theta}^{k} \partial_{t} P \pm \partial_{\theta}^{k+1} P\right)^{2}+e^{2 P}\left(\partial_{\theta}^{k} \partial_{t} Q \pm \partial_{\theta}^{k+1} Q\right)^{2}\right] \\
E_{k}(t) & =\sum_{ \pm}\left\|\mathcal{A}_{k, \pm}(t, \cdot)\right\|_{C^{0}\left(S^{1}, \mathbb{R}\right)} .
\end{aligned}
$$

Let us make the inductive assumption that

$$
E_{m}^{1 / 2}(t) \leq C_{m}(\ln t)^{m}
$$

for $m=0, \ldots, k-1$ and $t \geq 2$. Observe that since (10) holds for $k=0$, this holds for $k=1$. Compute, for $k \geq 1$,

$$
\left(\partial_{t} \mp \partial_{\theta}\right) \mathcal{A}_{k, \pm}=I_{1, k, \pm}+I_{2, k, \pm}
$$

where

$$
\begin{aligned}
I_{1, k, \pm}= & \frac{1}{2}\left\{-\left(\partial_{\theta}^{k} P_{t}\right)^{2}+\left(\partial_{\theta}^{k} P_{\theta}\right)^{2}+e^{2 P}\left[-\left(\partial_{\theta}^{k} Q_{t}\right)^{2}+\left(\partial_{\theta}^{k} Q_{\theta}\right)\right]\right\} \\
& -t e^{2 P}\left(P_{t} \pm P_{\theta}\right)\left[\left(\partial_{\theta}^{k} Q_{t}\right)^{2}-\left(\partial_{\theta}^{k} Q_{\theta}\right)^{2}\right] \\
& +t e^{2 P}\left(Q_{t} \pm Q_{\theta}\right)\left[\left(\partial_{\theta}^{k} Q_{t} \mp \partial_{\theta}^{k} Q_{\theta}\right)\left(\partial_{\theta}^{k} P_{t} \pm \partial_{\theta}^{k} P_{\theta}\right)\right. \\
& \left.-\left(\partial_{\theta}^{k} Q_{t} \pm \partial_{\theta}^{k} Q_{\theta}\right)\left(\partial_{\theta}^{k} P_{t} \mp \partial_{\theta}^{k} P_{\theta}\right)\right]
\end{aligned}
$$

and

$$
\begin{aligned}
I_{2, k, \pm}= & t\left\{\partial_{\theta}^{k}\left[e^{2 P}\left(Q_{t}^{2}-Q_{\theta}^{2}\right)\right]-2 e^{2 P}\left(Q_{t} \partial_{\theta}^{k} Q_{t}-Q_{\theta} \partial_{\theta}^{k} Q_{\theta}\right)\right\}\left(\partial_{\theta}^{k} P_{t} \pm \partial_{\theta}^{k} P_{\theta}\right) \\
& -2 t e^{2 P} \sum_{j=1}^{k-1}\left(\begin{array}{c}
k \\
j
\end{array}\right)\left[\partial_{\theta}^{j} P_{t} \partial_{\theta}^{k-j} Q_{t}-\partial_{\theta}^{j} P_{\theta} \partial_{\theta}^{k-j} Q_{\theta}\right]\left(\partial_{\theta}^{k} Q_{t} \pm \partial_{\theta}^{k} Q_{\theta}\right) .
\end{aligned}
$$

Fix $\theta$ and define $\gamma_{ \pm}(u)=(u, \theta \pm u)$. For $f: \mathbb{R}_{+} \times S^{1} \rightarrow \mathbb{R}$, let $f_{ \pm}=f \circ \gamma_{ \pm}$. Note that

Compute

$$
\partial_{u} f_{ \pm}=\left[\left(\partial_{t} \pm \partial_{\theta}\right) f\right]_{ \pm} \cdot
$$

$$
\mathcal{A}_{k, \pm}\left[\gamma_{\mp}(u)\right]=\mathcal{A}_{k, \pm}\left[\gamma_{\mp}\left(u_{0}\right)\right]+\int_{u_{0}}^{u}\left[\left(\partial_{t} \mp \partial_{\theta}\right) \mathcal{A}_{k, \pm}\right]_{\mp}(t) d t .
$$


Note that we have (13) and that each of the terms in $I_{1, k, \pm} \circ \gamma_{\mp}$ can be written, disregarding numerical factors, as a sum of terms of the form

$$
f_{1 \mp} f_{2 \mp} \partial_{u} f_{3 \mp} \text {. }
$$

Here, the possibilities for $f_{1}$ are

$$
1, \quad e^{2 P}, u e^{2 P}\left(P_{u} \pm P_{\theta}\right), \quad u e^{2 P}\left(Q_{u} \pm Q_{\theta}\right),
$$

the corresponding estimates for $\left|f_{1}\right|$ and $\left|\partial_{u} f_{1 \mp}\right|$ being, respectively,

$$
\text { 1, } C e^{2 P}, C u^{1 / 2} e^{2 P}, C u^{1 / 2} e^{P} \text { and } 0, C u^{-1 / 2} e^{2 P_{\mp}}, C e^{2 P_{\mp}}, C e^{P_{\mp}},
$$

where we have used (6)-(7) and the fact that (10) holds for $k=0$. The possibilities for $f_{2}$ are

$$
\left(\partial_{u} \pm \partial_{\theta}\right) \partial_{\theta}^{k} P, \quad\left(\partial_{u} \pm \partial_{\theta}\right) \partial_{\theta}^{k} Q
$$

the corresponding estimates for $\left|f_{2}\right|$ and $\left|\partial_{u} f_{2 \mp}\right|$ being, respectively

$$
u^{-1 / 2} E_{k}^{1 / 2}, u^{-1 / 2} e^{-P} E_{k}^{1 / 2} \text { and } u^{-1} E_{k}^{1 / 2}+\frac{(\ln u)^{k}}{u}, e^{-P_{\mp}}\left[u^{-1} E_{k}^{1 / 2}+\frac{(\ln u)^{k}}{u}\right]
$$

up to numerical factors. The reason for the latter is that

$$
\partial_{u}\left[\left(\partial_{u} \pm \partial_{\theta}\right) \partial_{\theta}^{k} P\right]_{\mp}=\left[\partial_{\theta}^{k}\left(P_{u u}-P_{\theta \theta}\right)\right]_{\mp}=\left\{-\frac{1}{u} \partial_{\theta}^{k} P_{t}+\partial_{\theta}^{k}\left[e^{2 P}\left(Q_{t}^{2}-Q_{\theta}^{2}\right)\right]\right\}_{\mp} .
$$

The first term on the right hand side satisfies a better estimate than the second to last expression in (17), and the terms resulting from the second term when at least one derivative hits the factor $e^{2 P}$ are also better. What remains to be considered are terms of the form

$$
\left[e^{2 P}\left(\partial_{\theta}^{j_{1}} Q_{t} \partial_{\theta}^{j_{2}} Q_{t}-\partial_{\theta}^{j_{1}} Q_{\theta} \partial_{\theta}^{j_{2}} Q_{\theta}\right)\right]_{\mp},
$$

where $j_{1}+j_{2}=k$. These terms can be estimated by the second to last expression in (17) due to the induction hypothesis. The argument for the second possibility for $f_{2}$ is similar. The possibilities for $f_{3}$ are $\partial_{\theta}^{k} P, \partial_{\theta}^{k} Q$, and the corresponding estimates for $\left|f_{3}\right|$ are

$$
\frac{(\ln u)^{k-1}}{u^{1 / 2}}, \quad e^{-P} \frac{(\ln u)^{k-1}}{u^{1 / 2}}
$$

due to the induction hypothesis (note that $k \geq 1$ ). Consider

$$
\int_{u_{0}}^{u} I_{1, k, \pm} \circ \gamma_{\mp}(t) d t
$$

Up to numerical factors, this integral can be written as a sum of terms of the form

$$
\int_{u_{0}}^{u} f_{1 \mp} f_{2 \mp} \partial_{t} f_{3 \mp} d t=\left[f_{1 \mp} f_{2 \mp} f_{3 \mp}\right]_{u_{0}}^{u}-\int_{u_{0}}^{u}\left[\partial_{t} f_{1 \mp} f_{2 \mp} f_{3 \mp}+f_{1 \mp} \partial_{t} f_{2 \mp} f_{3 \mp}\right] d t .
$$

Note that not all combinations occur and that when taking the products, all factors of $e^{P}$ in the estimates cancel. Using the definition of $I_{1, k, \pm}$ and the estimates written down above, we get

$$
\begin{aligned}
\left|\int_{u_{0}}^{u} I_{1, k, \pm} \circ \gamma_{\mp}(t) d t\right| \leq & C+C \frac{(\ln u)^{k-1}}{u^{1 / 2}} E_{k}^{1 / 2}(u) \\
& +C \int_{u_{0}}^{u}\left[\frac{(\ln t)^{k-1}}{t} E_{k}^{1 / 2}(t)+\frac{(\ln t)^{2 k-1}}{t}\right] d t .
\end{aligned}
$$


Let us turn to $I_{2, k, \pm}$. Up to numerical factors, the first term can be written as a sum of terms of the form

$$
t \partial_{\theta}^{j_{1}} P \cdots \partial_{\theta}^{j_{l}} P e^{2 P}\left(\partial_{\theta}^{m_{1}} Q_{t} \partial_{\theta}^{m_{2}} Q_{t}-\partial_{\theta}^{m_{1}} Q_{\theta} \partial_{\theta}^{m_{2}} Q_{\theta}\right)\left(\partial_{\theta}^{k} P_{t} \pm \partial_{\theta}^{k} P_{\theta}\right)
$$

where $j_{i} \geq 1, m_{i} \leq k-1$ and $j_{1}+\cdots+j_{l}+m_{1}+m_{2}=k$. Using the induction hypothesis, this can be estimated by

$$
C \frac{(\ln t)^{k-l}}{t^{(l+1) / 2}} E_{k}^{1 / 2}(t) .
$$

If $l \geq 1$, this estimate is as good as what we already have, so let us consider terms of the form

$$
t e^{2 P}\left(\partial_{\theta}^{m_{1}} Q_{t} \partial_{\theta}^{m_{2}} Q_{t}-\partial_{\theta}^{m_{1}} Q_{\theta} \partial_{\theta}^{m_{2}} Q_{\theta}\right)\left(\partial_{\theta}^{k} P_{t} \pm \partial_{\theta}^{k} P_{\theta}\right),
$$

where $m_{1}+m_{2}=k$ but $m_{i} \leq k-1$. Note that

$$
\begin{aligned}
\partial_{\theta}^{m_{1}} Q_{t} \partial_{\theta}^{m_{2}} Q_{t}-\partial_{\theta}^{m_{1}} Q_{\theta} \partial_{\theta}^{m_{2}} Q_{\theta}= & \frac{1}{2}\left[\left(\partial_{\theta}^{m_{1}} Q_{t} \pm \partial_{\theta}^{m_{1}} Q_{\theta}\right)\left(\partial_{\theta}^{m_{2}} Q_{t} \mp \partial_{\theta}^{m_{2}} Q_{\theta}\right)\right. \\
& \left.+\left(\partial_{\theta}^{m_{1}} Q_{t} \mp \partial_{\theta}^{m_{1}} Q_{\theta}\right)\left(\partial_{\theta}^{m_{2}} Q_{t} \pm \partial_{\theta}^{m_{2}} Q_{\theta}\right)\right]
\end{aligned}
$$

In other words, we need only concern ourselves with terms of the form

$$
t e^{2 P}\left(\partial_{\theta}^{m_{1}} Q_{t} \pm \partial_{\theta}^{m_{1}} Q_{\theta}\right)\left(\partial_{\theta}^{m_{2}} Q_{t} \mp \partial_{\theta}^{m_{2}} Q_{\theta}\right)\left(\partial_{\theta}^{k} P_{t} \pm \partial_{\theta}^{k} P_{\theta}\right) .
$$

We can then argue as before, with $f_{1}=t e^{2 P}\left(\partial_{\theta}^{m_{1}} Q_{t} \pm \partial_{\theta}^{m_{1}} Q_{\theta}\right), f_{2}=\left(\partial_{\theta}^{k} P_{t} \pm \partial_{\theta}^{k} P_{\theta}\right)$ and $f_{3}=\partial_{\theta}^{m_{2}} Q$. Note that since $m_{1}+m_{2}=k$ and $m_{i} \leq k-1$, we have $m_{i} \geq 1$. The arguments for the remaining terms in $I_{2, k, \pm}$ are similar, and by (13) we get

$$
\begin{aligned}
\int_{u_{0}}^{u}\left[\left(\partial_{t} \mp \partial_{\theta}\right) \mathcal{A}_{k, \pm}\right]_{\mp}(t) d t \leq & C+C \frac{(\ln u)^{k-1}}{u^{1 / 2}} E_{k}^{1 / 2}(u) \\
& +C \int_{u_{0}}^{u}\left[\frac{(\ln t)^{k-1}}{t} E_{k}^{1 / 2}(t)+\frac{(\ln t)^{2 k-1}}{t}\right] d t .
\end{aligned}
$$

Taking the supremum of the right hand side in (14), we thus get

$$
\begin{aligned}
\mathcal{A}_{k, \pm}\left[\gamma_{\mp}(u)\right] \leq & \left\|\mathcal{A}_{k, \pm}\left(u_{0}, \cdot\right)\right\|_{C^{0}\left(S^{1}, \mathbb{R}\right)}+C+C \frac{(\ln u)^{k-1}}{u^{1 / 2}} E_{k}^{1 / 2}(u) \\
& +C \int_{u_{0}}^{u}\left[\frac{(\ln t)^{k-1}}{t} E_{k}^{1 / 2}(t)+\frac{(\ln t)^{2 k-1}}{t}\right] d t .
\end{aligned}
$$

Taking the supremum of the left hand side (note that there is a $\theta$ hidden in $\gamma_{ \pm}$) and adding the two estimates, we get

$$
\begin{aligned}
E_{k}(u) \leq & C+C \frac{(\ln u)^{k-1}}{u^{1 / 2}} E_{k}^{1 / 2}(u) \\
+ & +C \int_{u_{0}}^{u}\left[\frac{(\ln t)^{k-1}}{t} E_{k}^{1 / 2}(t)+\frac{(\ln t)^{2 k-1}}{t}\right] d t
\end{aligned}
$$

Note that

Defining

$$
C \frac{(\ln u)^{k-1}}{u^{1 / 2}} E_{k}^{1 / 2}(u) \leq \frac{1}{2} C^{2} \frac{(\ln u)^{2 k-2}}{u}+\frac{1}{2} E_{k}(u)
$$

$$
\hat{E}_{k}(u)=E_{k}(u)+(\ln u)^{2 k},
$$


we thus get the estimate

$$
\begin{aligned}
\hat{E}_{k}(u) & \leq C+C \int_{u_{0}}^{u}\left[\frac{(\ln t)^{k-1}}{t} E_{k}^{1 / 2}(t)+\frac{(\ln t)^{2 k-1}}{t}\right] d t \\
& \leq C+C \int_{u_{0}}^{u} \frac{(\ln t)^{k-1}}{t} \hat{E}_{k}^{1 / 2}(t) d t
\end{aligned}
$$

By a Grönwall's lemma type argument, we conclude that

$$
\hat{E}_{k}(u) \leq C_{k}(\ln u)^{2 k}
$$

for $u \geq u_{0}$. This completes the induction proof.

Before we come to the curvature estimate, let us define

$$
\tau\left(t, t_{0}\right)=\sup _{\gamma} \int_{t_{0}}^{t}\left[-\left\langle\gamma^{\prime}(s), \gamma^{\prime}(s)\right\rangle\right]^{1 / 2} d s,
$$

where the supremum is taken over smooth timelike curves $\gamma(s)=[s, x(s)]$ where $x$ takes values on $T^{3}$. Note that for an arbitrary smooth timelike curve joining the hypersurface corresponding to $t_{0}$ with the hypersurface corresponding to $t$, one can change the parameterization so that it is of the above mentioned form.

Proposition 3. Consider a metric of the form (5), where $P, Q$ and $\lambda$ satisfy (6)(9). Assume furthermore that $P$ and $Q$ are not both independent of $\theta$ for all $t$. Given $t_{0}>0$ there are positive constants $c\left(t_{0}\right)$ and $C\left(t_{0}\right)$ such that for $t \geq t_{0}+1$,

$$
c\left(t_{0}\right) t^{-1 / 4} e^{\langle\lambda\rangle(t) / 4} \leq \tau\left(t, t_{0}\right) \leq C\left(t_{0}\right) t^{-1 / 4} e^{\langle\lambda\rangle(t) / 4} .
$$

Proof. Note that since (10) holds for $k=0,\left|\lambda_{\theta}\right|$ is bounded to the future, and consequently,

$$
|\lambda(t, \theta)-\langle\lambda\rangle(t)| \leq C\left(t_{0}\right)
$$

for $t \geq t_{0}$. Let us estimate

$$
\begin{aligned}
& t^{1 / 4} e^{-\langle\lambda\rangle(t) / 4} \int_{t_{0}}^{t}\left[-\left\langle\gamma^{\prime}(s), \gamma^{\prime}(s)\right\rangle\right]^{1 / 2} d s \\
& \leq \int_{t_{0}}^{t}\left(\frac{t}{s}\right)^{1 / 4} \exp \{[\lambda(s, \theta(s))-\langle\lambda\rangle(t)] / 4\} d s \\
& \leq C\left(t_{0}\right) \int_{t_{0}}^{t}\left(\frac{t}{s}\right)^{1 / 4} \exp \{[\langle\lambda\rangle(s)-\langle\lambda\rangle(t)] / 4\} d s .
\end{aligned}
$$

However, by Theorem 1.6 of [12] we have

$$
\left|\left\langle\lambda_{t}\right\rangle(t)-c_{0}\right| \leq C\left(t_{0}\right) t^{-1}
$$

for $t \geq t_{0}$, where $c_{0}>0$, assuming the solution is not independent of $\theta$. Thus

$$
\langle\lambda\rangle(s)-\langle\lambda\rangle(t) \leq-c_{0}(t-s)+C\left(t_{0}\right) \ln \frac{t}{s}
$$


We conclude that

$$
\begin{aligned}
& t^{1 / 4} e^{-\langle\lambda\rangle(t) / 4} \int_{t_{0}}^{t}\left[-\left\langle\gamma^{\prime}(s), \gamma^{\prime}(s)\right\rangle\right]^{1 / 2} d s \\
& \leq C\left(t_{0}\right) \int_{t_{0}}^{t}\left(\frac{t}{s}\right)^{\alpha\left(t_{0}\right)} \exp \left[-c_{0}(t-s) / 4\right] d s \\
& \quad=C\left(t_{0}\right) \int_{t_{0} / t}^{1} u^{-\alpha\left(t_{0}\right)} \exp \left[-c_{0} t(1-u) / 4\right] t d u
\end{aligned}
$$

If $t \leq 2 t_{0}$, this integral is bounded. If $t \geq 2 t_{0}$ we can divide the integral into two parts. Let us estimate

$$
\int_{1 / 2}^{1} u^{-\alpha\left(t_{0}\right)} \exp \left[-c_{0} t(1-u) / 4\right] t d u \leq 2^{\alpha\left(t_{0}\right)} \int_{1 / 2}^{1} \exp \left[-c_{0} t(1-u) / 4\right] t d u \leq \frac{4}{c_{0}} 2^{\alpha\left(t_{0}\right)}
$$

We also have

$$
\int_{t_{0} / t}^{1 / 2} u^{-\alpha\left(t_{0}\right)} \exp \left[-c_{0} t(1-u) / 4\right] t d u \leq \frac{4}{c_{0}}\left(\frac{t}{t_{0}}\right)^{\alpha\left(t_{0}\right)} \exp \left[-c_{0} t / 8\right]
$$

which is bounded by a constant depending on $t_{0}$. Note that the constants involved in the arguments above are independent of the curve $\gamma$. Thus

$$
\tau\left(t, t_{0}\right) \leq C\left(t_{0}\right) t^{-1 / 4} e^{\langle\lambda\rangle(t) / 4} .
$$

In order to get the opposite inequality, consider the curve $\gamma(s)=\left(s, x_{0}\right)$ where $x_{0}$ is a fixed point on $T^{3}$. We get

$$
\begin{aligned}
t^{1 / 4} e^{-\langle\lambda\rangle(t) / 4} \int_{t_{0}}^{t}\left[-\left\langle\gamma^{\prime}(s), \gamma^{\prime}(s)\right\rangle\right]^{1 / 2} d s & =\int_{t_{0}}^{t}\left(\frac{t}{s}\right)^{1 / 4} \exp \left\{\left[\lambda\left(s, \theta_{0}\right)-\langle\lambda\rangle(t)\right] / 4\right\} d s \\
& \geq c\left(t_{0}\right) \int_{t_{0}}^{t} \exp \{[\langle\lambda\rangle(s)-\langle\lambda\rangle(t)] / 4\} d s
\end{aligned}
$$

where $c\left(t_{0}\right)$ is a positive constant. Assuming $t \geq t_{0}+1$, we can use $(21)$ to prove that

$$
\begin{aligned}
\int_{t_{0}}^{t} \exp \{[\langle\lambda\rangle(s)-\langle\lambda\rangle(t)] / 4\} d s & \geq \int_{t-1 / 2}^{t} \exp \{[\langle\lambda\rangle(s)-\langle\lambda\rangle(t)] / 4\} d s \\
& \geq c\left(t_{0}\right)>0
\end{aligned}
$$

The proposition follows.

Proof of Theorem 2. Note that there is no loss of generality in choosing the vectors orthogonal to $e_{0}$ to be

$$
e_{1}=t^{1 / 4} e^{-\lambda / 4} \partial_{\theta}, \quad e_{2}=t^{-1 / 2} e^{-P / 2} \partial_{\sigma}, \quad e_{3}=t^{-1 / 2} e^{P / 2}\left(-Q \partial_{\sigma}+\partial_{\delta}\right) .
$$

It will be convenient to introduce the notation $\phi=t^{1 / 4} e^{-\lambda / 4}$. Note that

$$
c\left(t_{0}\right) \leq \phi(t, \theta) \tau\left(t_{0}, t\right) \leq C\left(t_{0}\right)
$$

for $t \geq t_{0}+1$ and $\theta \in S^{1}$ due to (20) and (19). Let $\Gamma_{\beta \gamma}^{\alpha} e_{\alpha}=\nabla_{e_{\beta}} e_{\gamma}$. Then

$$
\begin{aligned}
\left\langle R_{e_{\mu} e_{\nu}} e_{\alpha}, e_{\beta}\right\rangle= & e_{\nu}\left(\Gamma_{\mu \alpha}^{\delta}\right) \eta_{\delta \beta}-e_{\mu}\left(\Gamma_{\nu \alpha}^{\delta}\right) \eta_{\delta \beta}+\Gamma_{\mu \alpha}^{\delta} \Gamma_{\nu \delta}^{\kappa} \eta_{\kappa \beta}-\Gamma_{\nu \alpha}^{\delta} \Gamma_{\mu \delta}^{\kappa} \eta_{\kappa \beta} \\
& +\eta_{\delta \beta} \gamma_{\mu \nu}^{\kappa} \Gamma_{\kappa \alpha}^{\delta},
\end{aligned}
$$

where $\eta$ is the Minkowski metric and where $\left[e_{\alpha}, e_{\beta}\right]=\gamma_{\alpha \beta}^{\kappa} e_{\kappa}$ defines $\gamma_{\mu \nu}^{\kappa}$. The above formulas indicate what sign conventions we are using. One can check that all the 
terms except $e_{\nu}\left(\Gamma_{\mu \alpha}^{\delta}\right) \eta_{\delta \beta}-e_{\mu}\left(\Gamma_{\nu \alpha}^{\delta}\right) \eta_{\delta \beta}$ can be estimated by $\phi^{2}$. Furthermore, due to the estimate (10), one sees that the only problem consists in second derivatives of $\lambda$. However, one can check that these derivatives only occur in the combination $\lambda_{t t}-\lambda_{\theta \theta}$ which is $O\left(t^{-1 / 2}\right)$ due to (10) and the equations. This proves that $|R| \leq C \phi^{2}$, which together with (22) proves (11).

Proof of Proposition 1. Using the notation of the previous proof, $f_{1}=\phi^{-2} \tau^{-2}\left(t_{0}, t\right)$. Due to $(22)$, we conclude that $f_{1}(\tau, \cdot)$ is bounded from above and from below by positive constants. Since $\lambda_{\theta}$ is bounded, due to (10) for $k=0, \partial_{\theta} f_{1}$ is bounded. The conclusions concerning $f_{1}$ follow. Note that if we had an estimate of the form (10) without the logarithms, $\partial_{\theta}^{k} \lambda$ would be bounded to the future for any $k \geq 1$, and consequently $f_{1}(t, \cdot)$ would be bounded in any $C^{k}$ norm for $t \geq t_{0}+1$. Due to the results of [12], $P$ does not grow faster than logarithmically and $Q$ does not go to infinity faster than polynomially. Combining this information with (10), we conclude that $\partial_{\theta}^{k} P$ converges to zero for any $k \geq 1$ and that $\partial_{\theta}^{k} Q$ does not grow faster than polynomially. Due to (19) and the fact that $\langle\lambda\rangle=c_{0} t+O(\ln t)$, where $c_{0}>0$, cf. (21), we conclude that for large $t, t$ and $\ln \left[1+\tau\left(t_{0}, t\right)\right]$ are equivalent. Adding these pieces together, we get the conclusions of the proposition.

\section{BIANCHI VIII}

In this section we prove Theorem 3 and Proposition 2. The results necessary in order to carry out the computations are all taken from [11]. However, we refer the reader to [10] and the appendices of [9] for more details on curvature computations in the current setting.

Proof of Theorem 3. Let $e_{0}=\partial_{t}$ and $e_{i}=\left(a_{i}\right)^{-1} e_{i}^{\prime}$ (no summation) for $i=1,2,3$, with terminology as in subsection 1.2. Let Greek indices range from 0 to 3 and Latin indices from 1 to 3 . Define $\left[e_{\alpha}, e_{\beta}\right]=\gamma_{\alpha \beta}^{\delta} e_{\delta}$. Due to the form (12) and the fact that $e_{i}^{\prime}$ is a canonical basis, we have $\gamma_{i j}^{0}=\gamma_{0 i}^{0}=0$. Furthermore, we can define $n, \theta$ and $k$ by

$$
\gamma_{i j}^{k}=\epsilon_{i j l} n^{l k}, \gamma_{0 j}^{i}=-\theta_{i j} \text { and } k\left(e_{i}, e_{j}\right)=\left\langle\nabla_{e_{i}} e_{0}, e_{j}\right\rangle .
$$

Then $n^{l k}$ is diagonal, and the diagonal components will be denoted by $n_{i}$. Furthermore $\theta_{i j}$ is diagonal, and coincides with $-k\left(e_{i}, e_{j}\right)$. In what follows, we shall raise and lower Latin indices with $\delta_{i j}$, and we shall consequently not be very careful when it comes to indices being upstairs or downstairs. Let $\theta$ denote the trace of $\theta_{i j}$ and let $\sigma_{i j}$ be the traceless part. Since $\theta$ is never zero in the case of Bianchi VIII, cf. Lemma 21.5 of [9], we can define

$$
\Sigma_{i j}=\frac{\sigma_{i j}}{\theta}, \quad N_{i}=\frac{n_{i}}{\theta}, \quad \Sigma_{+}=\frac{3}{2}\left(\Sigma_{22}+\Sigma_{33}\right), \quad \Sigma_{-}=\frac{\sqrt{3}}{2}\left(\Sigma_{22}-\Sigma_{33}\right) .
$$

The relevant curvature quantities can be written

$$
\kappa=R_{\alpha \beta \gamma \delta} R^{\alpha \beta \gamma \delta}=8\left(E_{i j} E^{i j}-H_{i j} H^{i j}\right), \quad|R|^{2}=8\left(E_{i j} E^{i j}+H_{i j} H^{i j}\right)
$$


where

$$
\begin{aligned}
E_{i j} & =\frac{1}{3} \theta \sigma_{i j}-\left(\sigma_{i}{ }^{k} \sigma_{k j}-\frac{1}{3} \sigma_{k l} \sigma^{k l} \delta_{i j}\right)+s_{i j} \\
H_{i j} & =-3 \sigma^{k}{ }_{(i} n_{j) k}+n_{k l} \sigma^{k l} \delta_{i j}+\frac{1}{2} \operatorname{tr}(n) \sigma_{i j} \\
s_{i j} & =b_{i j}-\frac{1}{3} \operatorname{tr}(b) \delta_{i j} \\
b_{i j} & =2 n_{i}{ }^{k} n_{k j}-\operatorname{tr}(n) n_{i j},
\end{aligned}
$$

cf. p. 19 and p. 40 of [15]. Note that $E_{i j}$ and $H_{i j}$ define diagonal traceless matrices. In order to relate these expressions to the variables defined above, it will be convenient to define $\tilde{H}_{i}=H_{i i} / \theta^{2}, \tilde{E}_{i}=E_{i i} / \theta^{2}$. Then

$$
\begin{aligned}
\tilde{H}_{1} & =N_{1} \Sigma_{+}+\frac{1}{\sqrt{3}}\left(N_{2}-N_{3}\right) \Sigma_{-} \\
\tilde{H}_{2} & =-\frac{1}{2} N_{2}\left(\Sigma_{+}+\sqrt{3} \Sigma_{-}\right)+\frac{1}{2}\left(N_{3}-N_{1}\right)\left(\Sigma_{+}-\frac{1}{\sqrt{3}} \Sigma_{-}\right) \\
\tilde{E}_{2}-\tilde{E}_{3} & =\frac{2}{3 \sqrt{3}} \Sigma_{-}\left(1-2 \Sigma_{+}\right)+\left(N_{2}-N_{3}\right)\left(N_{2}+N_{3}-N_{1}\right) \\
\tilde{E}_{2}+\tilde{E}_{3} & =\frac{2}{9} \Sigma_{+}\left(1+\Sigma_{+}\right)-\frac{2}{9} \Sigma_{-}^{2}-\frac{2}{3} N_{1}^{2}+\frac{1}{3}\left(N_{2}-N_{3}\right)^{2}+\frac{1}{3} N_{1}\left(N_{2}+N_{3}\right) .
\end{aligned}
$$

Note that all other components of $\tilde{E}_{i}$ and $\tilde{H}_{i}$ can be computed from this due to the fact that $E_{i j}$ and $H_{i j}$ both define traceless matrices.

Let us consider the case when the initial data are of NUT type. The relevant statements concerning the asymptotics are then to be found on pp. 1955-1956 of [11]. In this case $\Sigma_{-}=0, N_{2}=N_{3}$ and

$$
\left|\Sigma_{+}-\frac{1}{2}\right|+\left|\left(N_{1} N_{2}\right)(\tau)+\frac{1}{4}\right|+\left|N_{2} e^{-3 \tau / 2}-c_{N}\right| \leq C e^{-3 \tau / 2}
$$

for some positive constants $c_{N}$ and $C$ and for $\tau \geq 0$. Furthermore, there are positive constants $c_{\theta}, C$ such that

for $\tau \geq 0$. Finally, $t$ and $\tau$ are related through

$$
\left|\frac{1}{\theta(\tau)}-c_{\theta} e^{3 \tau / 2}\right| \leq C
$$

$$
\left|t(\tau)-2 c_{\theta} e^{3 \tau / 2}\right| \leq C(1+\tau)
$$

for all $\tau \geq 0$. We conclude that $\tilde{H}_{i}$ and $\tilde{E}_{i}$ are all $O\left(e^{-3 \tau / 2}\right)=O(\theta)$. We conclude that $|R|^{2}=O\left(\theta^{6}\right)=O\left(t^{-6}\right)$. This proves the upper bound in the theorem. In order to prove the lower bound, we need only observe that

$$
\lim _{t \rightarrow \infty} t \tilde{H}_{1}=-\frac{c_{\theta}}{4 c_{N}} \neq 0 .
$$

Let us consider the general case. The necessary information is contained in Proposition 6, Corollary 7 and Corollary 8 of [11]. Note that in these results,

We have

$$
h:=\Sigma_{-}^{2}+\frac{3}{4}\left(N_{2}-N_{3}\right)^{2}, \quad v:=-N_{1}\left(N_{2}+N_{3}\right)-\frac{1}{2}, \quad u:=\Sigma_{+}-\frac{1}{2} .
$$

$$
\Sigma_{-}^{2}+\frac{3}{4}\left(N_{2}-N_{3}\right)^{2}=\frac{1}{4 \tau}+O\left(\frac{\ln \tau}{\tau^{2}}\right), \quad \Sigma_{+}=\frac{1}{2}+O\left(\tau^{-1}\right)
$$


and

$$
N_{1}\left(N_{2}+N_{3}\right)=-\frac{1}{2}+O\left(\tau^{-2}\right)
$$

By (82) of [11], we also have

$$
N_{2}=c_{N} \tau^{-3 / 4} e^{3 \tau / 2}\left[1+O\left(\frac{\ln \tau}{\tau}\right)\right]
$$

for some positive constant $c_{N}$. In combination with the above equations, this proves that $N_{1}$ converges to zero exponentially. In view of the above equations, we have

$$
\begin{aligned}
\tilde{H}_{1} & =O\left(\tau^{-1}\right), \\
\tilde{H}_{2} & =-\frac{1}{2} N_{2}\left(\Sigma_{+}+\sqrt{3} \Sigma_{-}\right)+\frac{1}{2} N_{2}\left(\Sigma_{+}-\frac{1}{\sqrt{3}} \Sigma_{-}\right)+O\left(\tau^{-1 / 2}\right) \\
& =-\frac{2}{\sqrt{3}} N_{2} \Sigma_{-}+O\left(\tau^{-1 / 2}\right), \\
\tilde{E}_{2}-\tilde{E}_{3} & =2 N_{2}\left(N_{2}-N_{3}\right)+O\left(\tau^{-1}\right) \\
\tilde{E}_{2}+\tilde{E}_{3} & =O\left(\tau^{-1}\right) .
\end{aligned}
$$

Thus

$$
\begin{aligned}
\theta^{-4}|R|^{2} & =8\left[\frac{3}{2}\left(\tilde{E}_{2}+\tilde{E}_{3}\right)^{2}+\frac{1}{2}\left(\tilde{E}_{2}-\tilde{E}_{3}\right)^{2}+\tilde{H}_{1}^{2}+\tilde{H}_{2}^{2}+\left(\tilde{H}_{1}+\tilde{H}_{2}\right)^{2}\right] \\
& =8\left[2 N_{2}^{2}\left(N_{2}-N_{3}\right)^{2}+\frac{8}{3} N_{2}^{2} \Sigma_{-}^{2}+N_{2} O\left(\tau^{-1}\right)\right] \\
& =\frac{64}{3} N_{2}^{2}\left[\Sigma_{-}^{2}+\frac{3}{4}\left(N_{2}-N_{3}\right)^{2}+N_{2}^{-1} O\left(\tau^{-1}\right)\right] .
\end{aligned}
$$

Taking (23) into account, we conclude that

$$
\lim _{\tau \rightarrow \infty} \tau N_{2}^{-2} \theta^{-4}|R|^{2}=\frac{16}{3} .
$$

On p. 1972 of [11], it is shown that there is a positive constant $\alpha_{\theta}$ such that

$$
\frac{1}{\theta}=\frac{\alpha_{\theta}}{\tau^{1 / 4}} e^{3 \tau / 2}\left[1+O\left(\frac{\ln \tau}{\tau}\right)\right], \quad t=\frac{2 \alpha_{\theta}}{\tau^{1 / 4}} e^{3 \tau / 2}\left[1+O\left(\frac{\ln \tau}{\tau}\right)\right] .
$$

Combining this with (24), we conclude that there are positive constants $c_{i}, i=$ $1,2,3$, such that

$$
\lim _{\tau \rightarrow \infty} t^{-2}(\tau) \tau N_{2}^{2}(\tau)=c_{1}, \quad \lim _{\tau \rightarrow \infty} t(\tau) \theta(\tau)=c_{2}, \quad \lim _{\tau \rightarrow \infty} \tau[\ln t(\tau)]^{-1}=c_{3} .
$$

Combining this with (25), we conclude that there is a positive constant $c_{0}$ such that

$$
\lim _{t \rightarrow \infty} t \ln t|R|(t)=c_{0} .
$$

Since there are sequences $\tau_{i, k} \rightarrow \infty, i=1,2$, such that $\Sigma_{-}\left(\tau_{1, k}\right)=0$ and $\left(N_{2}-\right.$ $\left.N_{3}\right)\left(\tau_{2, k}\right)=0$, cf. [11], the conclusions concerning the Kretschmann scalar follow by similar arguments.

Proof of Proposition 2. Let Ric denote the Ricci curvature of a spatial hypersurface of homogeneity. One can compute that

$$
\operatorname{Ric}\left(e_{i}, e_{j}\right)=2 n_{i k} n_{j}^{k}-\operatorname{tr}(n) n_{i j}-n^{k l} n_{k l} \delta_{i j}+\frac{1}{2}[\operatorname{tr}(n)]^{2} \delta_{i j},
$$


with terminology as in the proof of Theorem 3. Let $R_{i}=\operatorname{Ric}\left(e_{i}, e_{i}\right)$. We get

$$
\theta^{-2} R_{1}=\frac{1}{2} N_{1}^{2}-\frac{1}{2}\left(N_{2}-N_{3}\right)^{2}, \quad \theta^{-2} R_{2}=\frac{1}{2} N_{2}^{2}-\frac{1}{2}\left(N_{1}-N_{3}\right)^{2}
$$

and similarly for $R_{3}$. We see that $\theta^{-2} R_{1}$ tends to zero and that

$$
\theta^{-2} R_{2}=\frac{1}{2}\left(N_{2}+N_{3}\right)\left(N_{2}-N_{3}\right)-\frac{1}{2} N_{1}^{2}+N_{1} N_{3}
$$

The statement concerning $R_{3}$ is similar. Note that there are time sequences $\tau_{i, k} \rightarrow$ $\infty, i=1,2$, such that

$$
\lim _{k \rightarrow \infty}\left(N_{2}-N_{3}\right)\left(\tau_{1, k}\right) \tau_{1, k}^{1 / 2}=c_{0}
$$

for some positive constant $c_{0}$, and such that $\left(N_{2}-N_{3}\right)\left(\tau_{2, k}\right)=0$. Once one has made the above observations, the argument is similar to the end of the proof of Theorem 3 .

\section{ACKNOWLEDGMENTS}

I am grateful to Michael Anderson for discussions that led to me considering these problems.

\section{REFERENCES}

[1] Anderson M 1997 Scalar curvature and geometrization conjectures for 3-manifolds, in Comparison Geometry, MSRI Publications, Cambridge University Press, vol. 30 49-82

[2] Anderson M 2001 On long-time evolution in general relativity and geometrization of 3manifolds Comm. Math. Phy. 222 533-67

[3] Andersson L and Moncrief V 2004 Future complete vacuum spacetimes The Einstein equations and the large scale behavior of gravitational fields, 299-330, Birkhäuser, Basel

[4] Choquet-Bruhat Y and Moncrief V 2001 Future Global in Time Einsteinian Spacetimes with U(1) Isometry Group Ann. Henri Poincaré 2 1001-64

[5] Choquet-Bruhat Y 2004 Future complete U(1) symmetric Einsteinian spacetimes, the unpolarized case. The Einstein equations and the large scale behavior of gravitational fields, 251-298, Birkhäuser, Basel

[6] Choquet-Bruhat Y and Cotsakis S 2002 Global hyperbolicity and completeness J. Geom. Phys. 43 345-50

[7] Fischer A and Moncrief V 2001 The reduced Einstein equations and the conformal volume collapse of 3-manifolds Class. Quantum Grav. $184493-515$

[8] Ringström H 2000 Curvature blow up in Bianchi VIII and IX vacuum spacetimes Class. Quantum Grav. 17 713-731

[9] Ringström H 2001 The Bianchi IX attractor Ann. Henri Poincaré 2 405-500

[10] Ringström H 2001 The future asymptotics of Bianchi VIII vacuum solutions Class. Quantum Grav. 18 3791-823

[11] Ringström H 2003 Future asymptotics expansions of Bianchi VIII vacuum metrics Class. Quantum Grav. 20 1943-89

[12] Ringström H 2004 On a wave map equation arising in General Relativity Comm. Pure Appl. Math. 57 657-703

[13] Ringström H 2005 Data at the moment of infinite expansion for polarized Gowdy Class. Quantum Grav. 22 No 9 1647-1653

[14] Scott P 1983 The geometries of 3-manifolds Bull. London Math. Soc. 15 401-87

[15] Wainwright J and Ellis G F R 1997 editors Dynamical Systems in Cosmology Cambridge University Press

Max-Planck-Institut für Gravitationsphysik, Am Mühlenberg 1, D-14476 Golm, GerMANY 